\title{
Different Types of Periodic Activities in a Calcium Oscillation Model
}

\author{
Li Yuanhua, Zhou Yi and Ji Quanbao* \\ Dept. of Math., Huainan Normal University, Huainan 232038, Anhui, P. R. China
}

Received: 12 Apr. 2013, Revised: 17 Aug. 2013, Accepted: 19 Aug. 2013

Published online: 1 Nov. 2013

\begin{abstract}
In this paper, a model proposed by Marhl et al. is considered to investigate the effect of several parameters on the calcium bursting oscillation behavior. Different types of bursting are presented. Fast-slow burster analysis and first return map are used to explain the mechanism of the four types of bursting. The results are instructive for understanding the role of these parameters played in complex dynamics in the Marhl-Haberichter calcium oscillation model.
\end{abstract}

Keywords: Calcium oscillation, bursting, fast-slow dynamics, return map.

\section{Introduction}

In excitable as well as in non-excitable cells, many processes, such as cell secretion and egg fertilization, are performed by the oscillatory changing of free cytosolic calcium concentration. Calcium oscillations were found experimentally in the 1980s [1]. A large number of experimental works have confirmed the significant role of bursting oscillations in cell signaling. Due to the importance of oscillations, several mathematical models were established in order to explain the mechanism [2-10].

The first model was established by Shen and Larter [5], and its functioning bases on two main mechanisms, i.e. the calcium-induced calcium release (CICR) and the inositol trisphosphate crosscoupling (ICC). A more detailed research in explaining the complex calcium oscillations in non-excitable cells have been given by Borghans et al. [6] and Houart et al. [7] followed. In this paper, we focus on effects of different parameters to study their physiological roles in generating complex $\mathrm{Ca}^{2+}$ oscillations. Another model demonstrating bursting oscillations was proposed by Kummer et al. [8], which incorporates the feedback inhibition on the initial agonist receptor complex by $\mathrm{Ca}^{2+}$ and activated phospholipase C (PLC), as well as receptor type-dependent self-enhanced behavior of the activated $\mathrm{G}_{a}$ subunit. In the present article, the Marhl-Haberichter
$\mathrm{Ca}^{2+}$ oscillation model $[2,3,9,10]$ is analyzed by using the so-called fast-slow burster analysis and first return map [11, 12]. The dynamic mechanism of different calcium oscillations in non-excitable cells has been extensively investigated. For more details and analysis about these results see Ref. [13].

\section{Materials and methods}

The mathematical model we used was proposed by Marhl et al. [2]. The model is described by the following differential equations:

$$
\begin{gathered}
\frac{d C a_{\mathrm{cyt}}}{d t}=J_{\mathrm{ch}}-J_{\text {pump }}+J_{\text {leak }}+J_{\text {out }}-J_{\text {in }}+J_{\mathrm{CaPr}}-J_{\mathrm{Pr}} \\
\frac{d C a_{\mathrm{er}}}{d t}=\frac{\beta_{\mathrm{er}}}{\rho_{\mathrm{er}}}\left(J_{\text {pump }}-J_{\mathrm{ch}}-J_{\text {leak }}\right) \\
\frac{d C a_{\mathrm{m}}}{d t}=\frac{\beta_{\mathrm{m}}}{\rho_{\mathrm{m}}}\left(J_{\text {in }}-J_{\text {out }}\right)
\end{gathered}
$$

where $J_{\mathrm{ch}}=k_{\mathrm{ch}} \frac{C a_{\mathrm{cyt}}^{2}}{C a_{\mathrm{cyt}}^{2}+K_{1}^{2}}\left(C a_{\mathrm{er}}-C a_{\mathrm{cyt}}\right), \operatorname{Pr}_{\mathrm{tot}}=\operatorname{Pr}+C a \operatorname{Pr}$, $J_{\text {pump }}=k_{\text {pump }} C a_{\text {cyt }}, J_{\mathrm{Pr}}=k_{+} C a_{\text {cyt }} \operatorname{Pr}, J_{\mathrm{CaPr}}=k_{-} C a \operatorname{Pr}$, $J_{\text {leak }}=k_{\text {leak }}\left(C a_{\mathrm{er}}-C a_{\mathrm{cyt}}\right), J_{\text {out }}=\left(k_{\mathrm{m}} \frac{C a_{\mathrm{cyt}}^{2}}{C a_{\mathrm{cyt}}^{2}+K_{1}^{2}}+k_{\mathrm{mit}}\right) C a_{\mathrm{m}}$,

\footnotetext{
*Corresponding author e-mail: jqb_2001@163.com
} 
$C a_{\mathrm{tot}}=C a_{\mathrm{cyt}}+\frac{\rho_{\mathrm{er}}}{\beta_{\mathrm{er}}} C a_{\mathrm{er}}+\frac{\rho_{\mathrm{m}}}{\beta_{\mathrm{m}}} C a_{\mathrm{m}}+C a \mathrm{Pr}$, $J_{\text {in }}=k_{\text {in }} \frac{C a_{\mathrm{cyt}}^{8}}{C a_{\mathrm{cyt}}^{8}+K_{2}^{8}}$.

Three variables in the system are: the free $\mathrm{Ca}^{2+}$ concentration in the cytosol $\left(C a_{c y t}\right)$, free $\mathrm{Ca}^{2+}$ concentration in the endoplasmic reticulum(ER) $\left(\mathrm{Ca}_{e r}\right)$, and the free $\mathrm{Ca}^{2+}$ concentration in the mitochondria $\left(C a_{m}\right)$.

Parameters for which all calculations are made if not otherwise stated: $k_{\text {leak }}=0.01 s^{-1}, \quad k_{\text {pump }}=20.0 s^{-1}$, $k_{\text {in }}=300 \mu M s^{-1}, \quad k_{\mathrm{m}}=125 s^{-1}, \quad k_{+}=0.09 \mu M s^{-1}$, $k_{-}=0.01 s^{-1}, K_{1}=5.0 \mu M, K_{2}=0.8 \mu M, C a_{\text {tot }}=90 \mu M$, $P r_{\mathrm{tot}}=120 \mu M, \rho_{\mathrm{er}}=0.01, \beta_{\mathrm{er}}=0.0025, \rho_{\mathrm{m}}=0.01$, $\rho_{\mathrm{m}}=0.0025$.

Equations (1)-(3) construct a full system with the fast subsystem (FS.) and the slow subsystem (SS.). In the fast-slow analysis, we determine the type of bursting by behavior of FS, where the slow variable $C a_{m}$ is considered as bifurcation parameter.

\section{Results}

When $k_{\mathrm{ch}}=1500$, there is a point-cycle bursting of subHopf-suHopf type, as shown in Fig.1 and 2. HB and LPC are subcritical Hopf bifurcation and fold limit cycle bifurcation respectively. Solid and doted lines (right and left side of HB) are stable and unstable steady state. Dash (thin solid) lines are stable (unstable) periodic solutions. The closed line represents the limit cycle trajectory of the complete system.

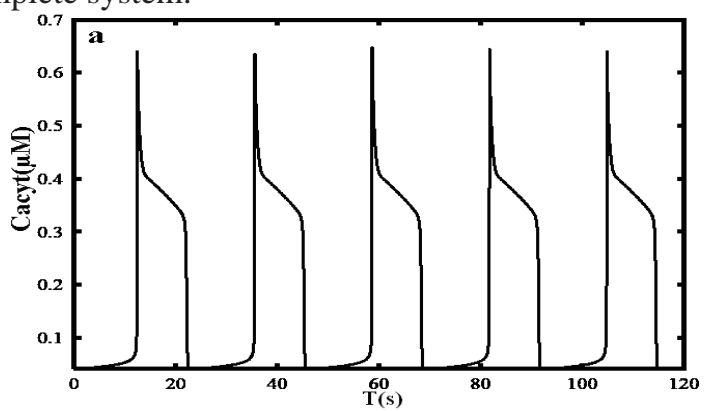

Fig. 1. Time series of $C a_{c y t}$ with $k_{c h}=1500$ of point-cycle bursting.

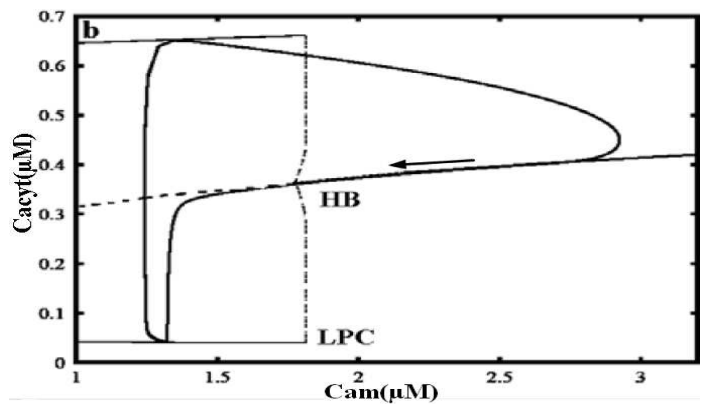

Fig. 2. Fast-slow dynamical analysis of point-cycle bursting.
The main character of this type is the active and silent phases of bursting depend on a stable steady state and a stable limit cycle related to a subHopf bifurcation at HB, see Fig.2. When the trajectory passes the subcritical Hopf bifurcation (HB), the stable steady state branch turns unstable. Therefore, the trajectory unfolds from the unstable steady state to stable periodic attractors. This starts the active phase of bursting. As time progresses the trajectory passes through the unstable limit cycle. Due to the attractive stable foci, the trajectory turns to the stable steady state on the other side of the subcritical Hopf bifurcation. The silent phase of bursting starts again.

When $k_{\mathrm{ch}}=2225$ and $k_{\text {leak }}=0.03$, this system has two attractors. The trajectory tends to one of them, depending on the different initial conditions. This phenomenon is called birhythmicity. For one initial condition $\left(C a_{\text {cyt }}=0.5, C a_{\mathrm{er}}=0.5, C a_{\mathrm{m}}=0.85\right)$, there is only regular spiking of $C a_{m}$ shown in Fig.3, so the trajectory is a simple unfolded limit cycle, as illustrated in Fig.4. However, for another initial condition $\left(C a_{\text {cyt }}=0.5, C a_{\mathrm{er}}=0.5, C a_{\mathrm{m}}=1\right)$ one period of oscillations of $C a_{m}$ composes of two spikes differed in the amplitude (as shown in Fig.5, which leads to a double folded limit cycle (see Fig.6. Bifurcation analysis is similar to the above and omitted here).

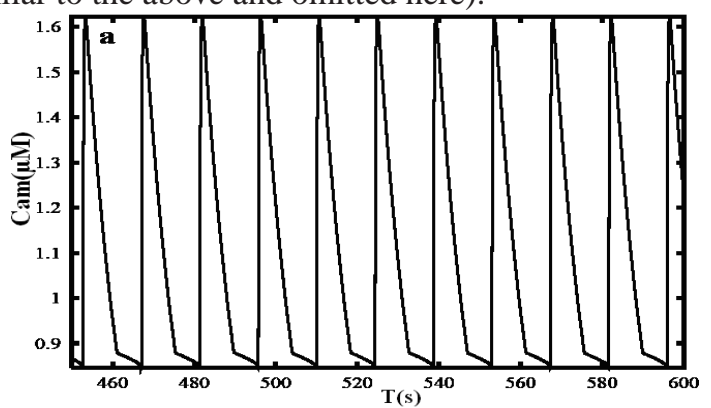

Fig. 3. Time series of birhythmic bursting with $k_{c h}=2225$ and $k_{\text {leak }}=0.03$.

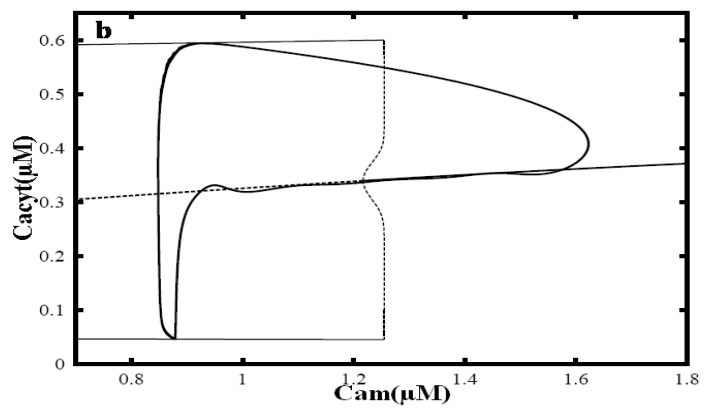

Fig. 4. Unfolded limit cycle trajectory of the complete system in $\left(C a_{c y t}, C a_{m}\right)$-plane. 


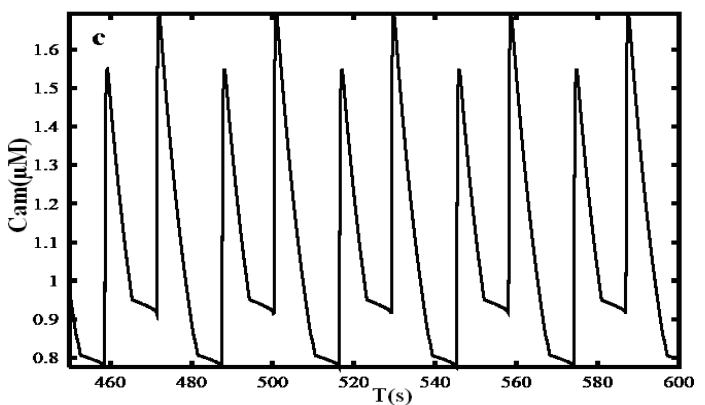

Fig. 5. Time series of $C a_{m}$ with $k_{c h}=2225$ and $k_{\text {leak }}=0.03$ for different initial conditions.

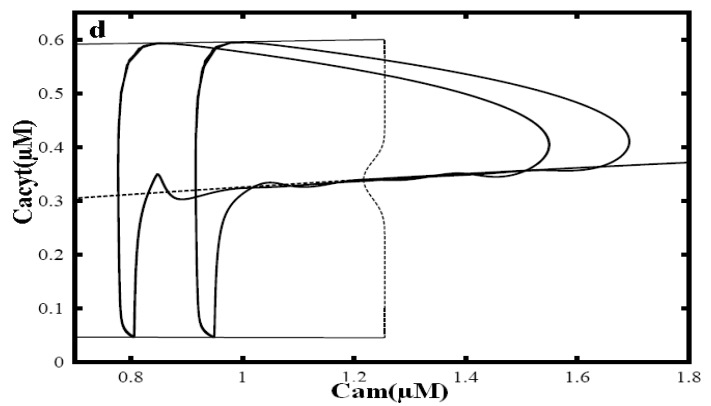

Fig. 6. Folded limit cycle trajectory of the complete system in $\left(\mathrm{Ca} a_{c y t}, \mathrm{Ca} a_{m}\right)$-plane.

When $k_{\mathrm{ch}}=2000$ and $k_{\text {pump }}=17.8$, the full system has four attractors. We call this phenomenon quadric-rhythmicity (see Fig.7). For some strict conditions, the trajectory of the complete system can show quadruple folded limit cycle (as shown in Fig.8). Fig.9 shows such a return map for an example of quadric-rhythmic bursting. In this figure, the successive maxima of $C a_{m}$ are plotted against their predecessors. Points of the bisector line represent cycles of constant amplitudes. Each single point of the return map represents one cycle of the system trajectory.

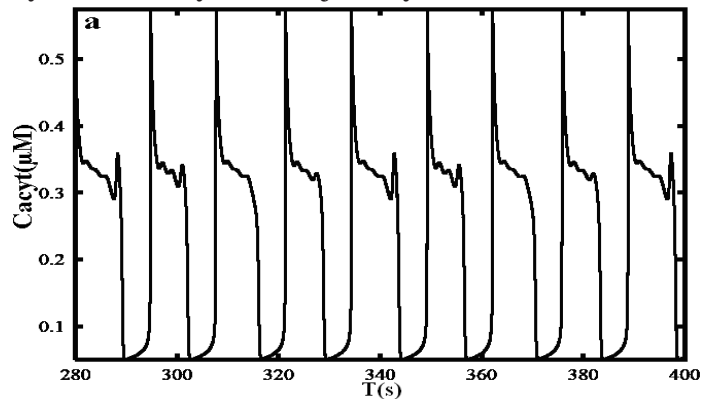

Fig. 7. Time series of quadric-rhythmic bursting with $k_{c h}=2000$ and $k_{\text {pump }}=17.8$.

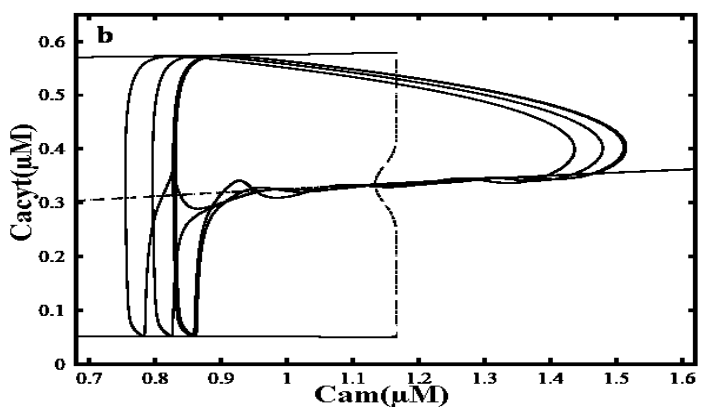

Fig. 8. Trajectory of the complete system in $\left(\mathrm{Ca}_{c y t}, \mathrm{Ca}\right)$-plane with $k_{c h}=2000$ and $k_{\text {pump }}=17.8$.

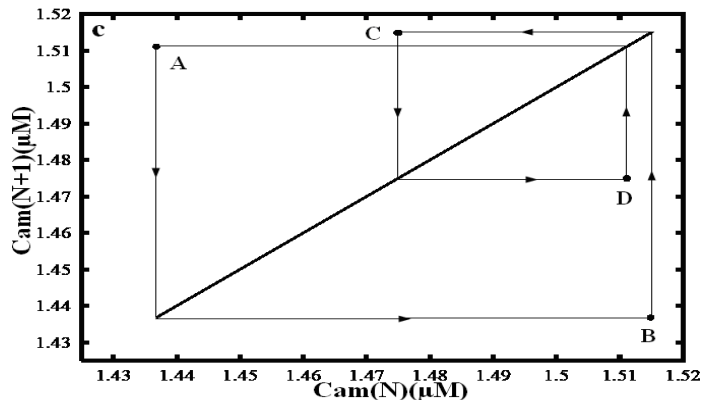

Fig. 9. Return map of $C a_{m}$ with $k_{c h}=2000$ and $k_{\text {pump }}=17.8$.

We start at the point A (marked by an arrow in the anti-clockwise direction), along the grey line, then we pass point $\mathrm{B}, \mathrm{C}$ and $\mathrm{D}$. At last we return to point $\mathrm{A}$. This is a strong evidence for quadric-rhythmic bursting.

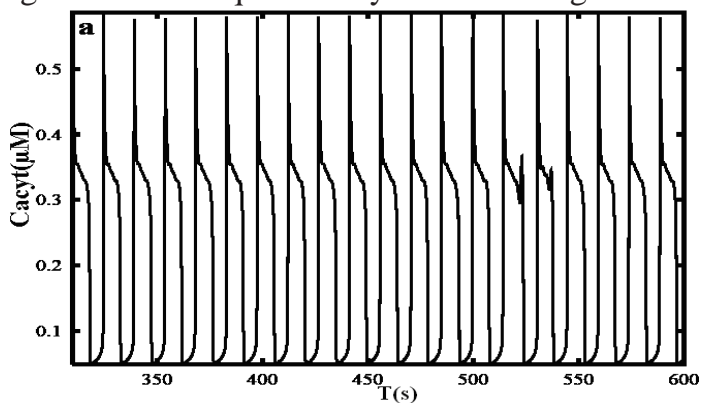

Fig. 10. Time series of chaotic bursting with $k_{c h}=1800$ and $k_{\text {pump }}=17.8$.

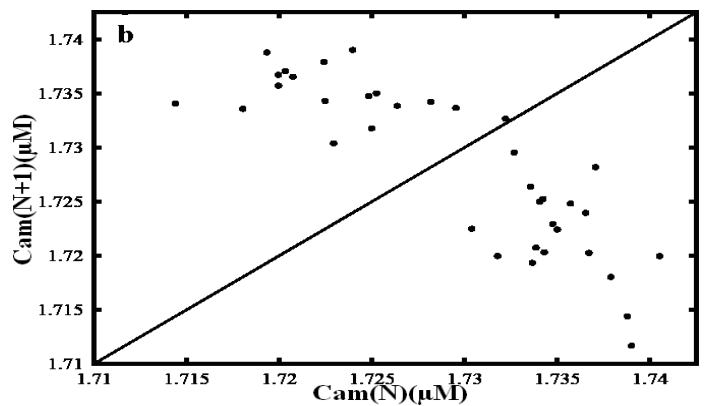

Fig. 11. Return map of $C a_{m}$ with $k_{c h}=1800$ and $k_{\text {pump }}=17.8$.

When $k_{\mathrm{ch}}=1800$ and $k_{\text {pump }}=17.8$, the full system displays a chaotic bursting. These chaotic behaviors are 
characterized by a positive value of the largest Lyapunov exponent and neglected here. For $k_{\mathrm{ch}}=1800$ and $k_{\text {pump }}=17.8$, the time course of chaos is shown in Fig.10. Note that the amplitudes of the $C a_{\text {cyt }}$ spikes keep almost constant all the time. The corresponding return map to the time series is presented in Fig.11. In this figure, the successive maxima of $C a_{m}$ are plotted against their predecessors. From this figure we can see that these points is very messy, which means the occurrence of chaos.

\section{Discussion}

In this paper, the effects of different parameters on the $\mathrm{Ca}^{2+}$ oscillations have been studied. Four types of bursting $\mathrm{Ca}^{2+}$ oscillations are given. Separately, we analyze point-cycle bursting of subHopf-subHopf type, birhythmicity, quadric-rhythmicity and chaos. Lots of studies show that frequency encoding and amplitude encoding play an extraordinary role in information processing and signal transduction in many biological systems. From this point of view, it is very important to analyze different types of bursting calcium oscillations, since different types of bursting behavior could reflect different encoding of biologically relevant information. Therefore, more types of bursting behavior should be found and further studies will be necessary to determine more precisely effects of different parameters on the $\mathrm{Ca}^{2+}$ oscillations.

\section{Acknowledgement}

This work is partially supported by the National Natural Science Foundation of China under Grant no. 11202083, the Natural Science Foundation of the Anhui Higher Education Institutions of China under Grant no. KJ2013A240, KJ2013Z309 and the Youth Foundation of Huainan Normal University (2012LK17).

\section{References}

[1] M. J. Berridge, Neuron, 21, 13-26 (1998).

[2] M. Marhl, T. Haberichterv and M. Brumen, Bio. Systems, 57, 75-86 (2000).

[3] M. Marhl, S. Schuster and M. Brumen, Biophys. Chem., 71, 125-132 (1998)

[4] J. P. Newman and R. J. Butra, Chaos, 20, 1-8 (2010).

[5] P. Shen and R. Larter, Biophys. Chem., 17, 225-232 (1995).

[6] J. M. Borghans, G. Dupont and A. Goldbeter, Biophys. Chem., 66, 25-41 (1997).

[7] G. Houart, G. Dupont and A. Goldbeter, Bursting, Bull. Math. Biol., 61, 507-530 (1999).

[8] U. Kummer, L. F. Olsen, C. J. Dixon, A. K. Green, E. Borhberg-Bauer and G. Baier, Biophys., 79, 1188-1195 (2000).
[9] T. Haberichter, M. Marhl and R. Heinrich, Biophys. Chem., 90, 17-30 (2001).

[10] M. Perc and M. Marhl, 18, 759-773 (2003).

[11] J. Rinzel, Bursting oscillations in an excitable membrane model. Lecture Notes in Mathematic, Springer-Verlag, 304316 (1985).

[12] P. Meng and Q. S. Lu, Chin. Phys. Lett., 27, 0105020010502 (2010).

[13] E. G. Roger and P. Mark, Society for Industrial and Applied Mathematics, 66, 1917-1948 (2006).

[14] E. Harvey, V. Kirk, M. Wechselberger and J. Sneyd, J. Nonlinear Sci., 21, 639-683 (2011).

[15] P. C. Rech, Dynamics in the parameter space of a neuron model, Chin. Phys. Lett., 29, 60506-060506 (2012).

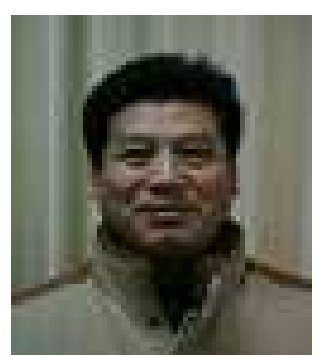

Yuanhua

Li graduated from Anhui Normal University in 1987. He is currently an associate professor in Huainan Normal University. His research interests are in the areas of differential equations and algebra.

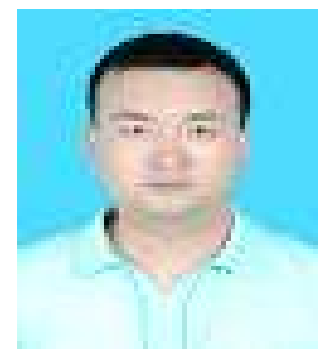

Yi Zhou received the MS degree in Mathematics from Anhui Normal University in 2012. He is currently an assistant in Huainan Normal University. His research interests are in the areas of differential equations, and nonlinear dynamics.

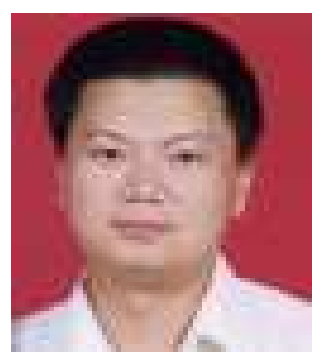

Quanbao Ji received the MS degree in Mathematics from Shanghai Normal University in 2006 and the $\mathrm{PhD}$ degree in Mechanical Engineering from Beihang University in 2009. He is currently an associate professor in Huainan Normal University. His research interests are in the areas of nonlinear dynamics and information systems. 